\title{
Plasma Imaging and Spectroscopy Diagnostics Developed on 100-500-kA Pulsed Power Devices
}

\author{
DANIEL B. SINARS, LEV GREGORIAN, DAVID A. HAMMER, FELLOW, IEEE, AND \\ YITZHAK MARON, MEMBER IEEE
}

\section{Invited Paper}

We discuss the development of high-resolution plasma imaging and spectroscopy diagnostics for the soft X-ray and ultraviolet energy ranges developed and used on 100-500 kA pulsed power facilities. Requiring just a few people to run and modest infrastructure investment, these facilities are cost-effective test beds for new ideas and technologies as well as for training students. Most of the diagnostics discussed here are presently or will soon be in use on larger scale facilities worldwide.

Keywords-Plasma pinch, ultraviolet (UV) spectroscopy, X-ray imaging, $X$-ray spectroscopy.

\section{INTRODUCTION}

High energy density plasma physics is replete with examples of large-scale facilities requiring several tens of people to run. This trend is driven by the increasingly large requirements for higher currents, radiation levels, laser light intensities, etc. Present examples of such facilities include the National Ignition Facility at Lawrence Livermore National Laboratories, Livermore, CA [1]; the Z facility at Sandia National Laboratories, Albuquerque, NM [2], the Angara-5-1 facility, Troitsk, Russia [3]; the Laser Megajoule laser facility, Bordeaux, France; and the OMEGA laser facility at the University of Rochester, Rochester, NY [4]. Such facilities are beyond the scope of a typical university research group.

Manuscript received October 16, 2003; revised January 27, 2004. This work was supported in part by Sandia National Laboratories, Albuquerque, NM, under Contract BD-9356, in part by the U.S. Department of Energy (DOE) under Grants DE-FG02-ER54496 and DE-FG03-98DP00217, in part by the German-Israeli Project Cooperation Foundation (DIP), and in part by the Israeli Science Foundation (ISF). The work of Sandia National Laboratories is supported by the DOE under Contract DE-AC04-94AL85000.

D. B. Sinars is with the Sandia National Laboratories, Albuquerque, NM 87185-1193 USA (e-mail: dbsinar@sandia.gov).

L. Gregorian and Y. Maron are with the Faculty of Physics, Weizmann Institute of Science, Rehovot 76100, Israel (e-mail: fngregor@ plasma-gate.weizmann.ac.il; fnmaron@wisemail.weizmann.ac.il).

D. A. Hammer is with the Laboratory of Plasma Studies, Cornell University, Ithaca, NY 14853 USA (e-mail: dah5@cornell.edu).

Digital Object Identifier 10.1109/JPROC.2004.829010
Under these circumstances, it may be imagined that university-scale facilities play only a minor role in driving the development of new ideas and technologies. This is far from being the case. One of the critical drawbacks of larger facilities is that by their nature the repetition rate tends to be very low and the cost of experiments (especially experiments requiring facility modifications) are very high. As a result, experimental campaigns are determined months to years ahead, which severely impacts the flexibility to try out new ideas. Smaller facilities can be used for such purposes with days to weeks of planning. This, along with the smaller number of people necessary to carry out an experiment, allows new ideas to be rapidly implemented, iterated, and refined on small-scale facilities.

This paper addresses the role that research groups working with modest-scale pulsed power facilities at universities have played and can play in the dense $Z$-pinch area by discussing recent examples of diagnostic development that have been carried out at Cornell University, Ithaca, NY, and the Weizmann Institute of Science, Rehovot, Israel. The research discussed here exemplifies the research that can be carried out using $10^{10}-10^{11}-\mathrm{W}$ university-scale pulsed power systems. In particular, we discuss high-resolution $\mathrm{X}$-ray backlighting, X-ray spectroscopy, and ultraviolet (UV) spectroscopy diagnostic development and use on our facilities. Laser-based imaging and visible spectroscopy diagnostics have also been important research tools for precision measurements in our laboratories as well as in other university pulsed power laboratories. However, we have chosen to concentrate on the X-ray and UV diagnostics, as their development is part of our current research effort. Although we emphasize our own work, we reference contributions to research being done elsewhere. We also point out how the work described here contributes to research being carried out and planned on larger scale facilities.

Dense $Z$ pinches driven by pulsed power generators are well known to be good sources of radiation [5]. The wave- 
length range depends on the plasma temperature achieved, which depends in turn on the generator peak current and how the plasma is made. Here we discuss plasmas produced by 220-450-kA peak current generators from gas puffs and exploding metal wires. Gas-puff $Z$-pinch systems are ideal for generating $10^{16}-10^{19}-\mathrm{cm}^{-3}$ plasmas with temperatures in the 10-20-eV range for the detailed study of plasma density, temperature, ionization state, and the magnetic field profile using UV spectroscopy [6], [7]. As such, novel methods for making such measurements can be developed that can be extended to plasmas on larger facilities. Exploding-wire $Z$ pinches are excellent for X-ray spectroscopy because the magnetically-driven, unstable implosion of the plasma produces extremely bright X-ray sources with temperatures that can reach into the kilovolt range using materials ranging from $\mathrm{Mg}$ to $\mathrm{W}$. To study these bright sources (known as micropinches) with high-resolution diagnostics, however, it was necessary to develop a method for localizing the X-ray source. This led to the development in the early 1980s of the $X$-pinch plasma [8], described in Section II. The study of micropinch plasmas using $X$ pinches remains today a fertile area of research accessible to universities.

The study of bright-spot production in $X$ pinches at Cornell University required the development and implementation of several advanced high-resolution X-ray diagnostics. One such diagnostic was the $X$ pinch itself. Taking advantage of the micropinch plasma X-ray sources localized at the center of the $X$ pinch, high-resolution point-projection X-ray backlighting images could be made of dense plasmas, such as exploding wires or other $X$ pinches [9]. Focusing X-ray spectroscopy diagnostics with one-dimensional (1-D) or two-dimensional (2-D) spatial resolution based on bent crystals, which were initially developed with high-intensity lasers during the 1970s [10], [11], were applied to the exploding-wire $X$ pinches at the 200-400 kA peak current level. By varying the source and detector positions in the bent-crystal spectroscopy geometry, monochromatic X-ray microscopy [12] and backlighting [13], [14] diagnostics were developed for imaging plasmas with $<10-\mu \mathrm{m}$ spatial resolution.

The diagnostics listed above are each described briefly in this paper, along with example implementations at larger pulsed power facilities. Section II describes the $X$-pinch $\mathrm{X}$-ray source and point-projection $\mathrm{X}$-ray backlighting diagnostics developed using this source. Section III presents the development and concept behind focusing X-ray spectrographs with 1-D or 2-D spatial resolution. Section IV describes monochromatic X-ray microscopy and backlighting diagnostics developed using a modification of the focusing spectrograph geometry. Section V discusses UV diagnostics and techniques developed to study gas-puff Z-pinch implosions. A summary is presented in Section VI along with some concluding remarks.

\section{X-Pinch Point-Projection BAcklighting}

\section{A. X-Pinch X-Ray Source}

An $X$ pinch is a variant of the single-wire $Z$ pinch that is made using two (or more) fine metal wires (typically 5-50 $\mu \mathrm{m}$ in diameter), which cross and touch at a single
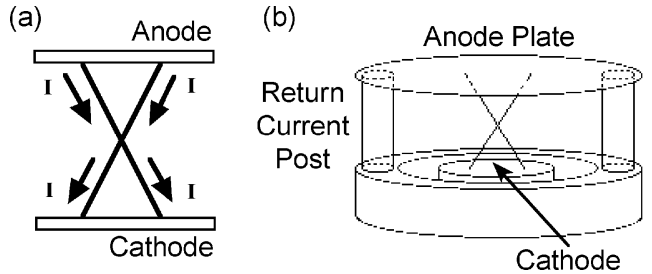

Fig. 1. (a) Diagram illustrating the concept of the $X$ pinch in its simplest form. Two wires are strung across an anode-cathode gap so that they make contact at a single point, forming an " $X$ " shape as shown. The current passing through the legs of the $X$ pinch is insufficient to form bright radiation sources. At the cross point of the $X$ pinch, where the currents from the legs add, a small $Z$ pinch forms that subsequently collapses and forms bright $X$-ray sources in the process. (b) A schematic diagram of a typical $X$-pinch setup in a pulsed power device. In some experiments, two or more $X$ pinches are driven in parallel, and series configurations are also possible.

point (forming an " $\mathrm{X}$ " shape). A current pulse from a pulsed power device (e.g., a 200-400-kA, 40-ns rise time, 100-ns full-width at half-maximum (FWHM) pulse) is passed through the wires, causing them to explode and form plasma. As shown in Fig. 1, the currents in the individual wires add at the cross point of the wires, where the total magnetic field is strong enough to magnetically implode the plasma, forming a $~ 300-\mu \mathrm{m}$-long, 100-150- $\mu \mathrm{m}$-diameter $Z$ pinch. The $Z$ pinch implodes unstably, forming small-diameter necks and, eventually, one or two micropinches. Soft $\mathrm{X}$-rays (1-10 keV) are emitted predominantly from these micropinches, thus assuring that such radiation is emitted from within a small region near the initial cross point of the $X$ pinch.

Experiments studying single-wire $Z$ pinches [15], [16] revealed small, localized plasma constrictions (micropinches) from which X-rays were emitted. These micropinches formed at random points along the wire-induced plasma. Thus, until recently, relatively little was known about these $\mathrm{X}$-ray sources because this random nature prevented the use of diagnostics with high spatial and/or temporal resolution to study them. The $X$ pinch, first proposed by Ulshmid in 1981 during a visit to the P. N. Lebedev Physical Institute, Moscow, Russia [8], avoids this spatial location problem. In fact, bright spots in $X$ pinches can be made to form at reproducible times (to within $\pm 1-3 \mathrm{~ns}$ ) as well as in a predetermined region of space (to within $\pm 0.1 \mathrm{~mm}$ ), which allows the use of high-precision diagnostics. In addition, the small size and predictable location of the X-ray source(s) make the $X$ pinch an attractive $\mathrm{X}$-ray source for numerous applications, such as various $X$-ray imaging configurations [9], [13], [14], microlithography [17]-[19], and precision spectroscopy [10], [20].

Time-integrated spectrographs with high spatial resolution (Section III) revealed that $X$-pinch bright spots are strong sources of both line and continuum radiation. However, while the continuum radiation is confined to the bright spots near the cross point of the $X$ pinch, the line radiation comes also from larger regions above and below the cross point [11], [21]. Experiments using monochromatic crystal microscopes with 2-D spatial resolution to look at line radiation emitted from $X$ pinches also saw that the line 


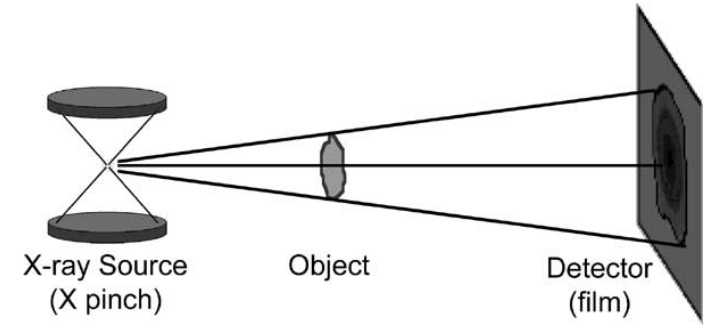

Fig. 2. Schematic diagram of the $X$-pinch point-projection radiography setup. The spectral bandpass used for radiography is generally determined by choosing an appropriate combination of films and filters.

radiation was emitted from relatively large regions of the $X$ pinch, though the bulk of it was usually concentrated at the cross point [14]. This is consistent with results from point-projection experiments using $X$ pinches, which obtain images with the highest spatial resolution only when the line radiation is cut out using filters [22].

More recently, experiments studying $X$ pinches at Cornell University have used diagnostics with micrometer-scale spatial resolution and/or $<10$-ps time resolution to study the properties of the brightest portions of the X-ray emitting plasmas [23], [24]. These experiments have concluded that the bright-spot plasmas have sizes as small as about $1 \mu \mathrm{m}$ diameter, electron temperatures $\sim 1 \mathrm{keV}$, and peak densities near solid density. These properties change rapidly over the 0.1-1-ns duration of the X-ray emission, and the radiation $>3 \mathrm{keV}$ tends to have an even shorter characteristic time $(10-100 \mathrm{ps})$ associated with it than the radiation $<3 \mathrm{keV}$.

The physical mechanisms responsible for the formation of the bright spots in $X$-pinch plasmas are not well understood. Computer simulations of the plasma dynamics have been underway for a few years, but their validity is suspect before the extreme conditions believed to be responsible for the X-ray bursts are reached [25], [26]. It is safe to say that additional experimental, theoretical, and computational work is required.

\section{B. Standard Point-Projection Imaging Using the X Pinch}

The small size of the bright $X$-ray emission regions from an $X$ pinch $(1-10 \mu \mathrm{m})$ make it an ideal source for point-projection radiography. In the point-projection geometry, depicted schematically in Fig. 2, the spatial resolution of the object $\sigma$ is given approximately by $\sigma=\delta(M-1) / M$, where $\delta$ is the diameter of the $\mathrm{X}$-ray source and $M$ is the magnification. The short (0.01-0.1-ns) duration of the $\mathrm{X}$-ray emission is also of great benefit, as the $X$ pinch can be used to radiograph fast-moving, dense plasmas without motional blurring. One of the earliest applications of $X$-pinch point-projection radiography was the study of fast current-driven single-wire explosions [9]. These early experiments established the presence of dense wire cores for extended periods [27].

The spatial resolution of the initial experiments was $\sim 10 \mu \mathrm{m}$, but subsequent work refined the parameters of the $X$ pinch to reduce the $\mathrm{X}$-ray source size, thereby achieving diffraction-limited spatial resolutions of $2-3 \mu \mathrm{m}$ even at extremely high magnifications, e.g., 90 [22]. These high-resolution images were obtained using Mo $X$-pinch $\mathrm{X}$-ray sources and Ti filters in front of the film pack. This combination restricts the imaging wavelengths to primarily the 3-5 keV range, with some contributions from $>8 \mathrm{keV}$. This photon energy range does not include Mo line radiation, which was observed to come from a larger spatial region than the continuum radiation. The study of $X$-pinch and single-wire explosions using these $\mathrm{X}$-ray sources continues, and sample radiographs from recent publications [28], [29] are shown in Fig. 3.

$X$-pinch point-projection radiography has recently been employed to study the early stages of the evolution of cylindrical wire-array implosions on larger pulsed power facilities, such as the 1-MA MAGPIE facility, Imperial College, London, U.K. [30] and the 3-5-MA Angara facility [3]. In these cases, the $X$ pinch is driven with current tapped from the main driver current. Recent work has demonstrated that independent, compact pulsed power drivers can be built to drive $X$-pinch implosions using as little as $50 \mathrm{kA}$ with a 40 -ns rise time pulse [31]. Such compact sources could enhance the usefulness of this backlighting source for facilities such as the 20-MA Z facility at Sandia, at which an independently timed X-ray source would be valuable.

\section{Phase-Contrast Imaging Using the $X$ Pinch}

If a spatially coherent $\mathrm{X}$-ray source is used in a point-projection imaging geometry with the detector placed far from the object, wave optics effects (refraction and diffraction) become important as depicted in Fig. 4. It has been noted that this can enable "phase-contrast" images to be made of objects that are otherwise too thin to be observed solely by X-ray absorption effects [32], [33]. For example, in some of the single-wire experiments described in Section II-B, the wires had thin plastic coatings. As the wires exploded, the plastic coating also exploded and formed a thin shell around the expanding wire core. An example image of such a plastic shell surrounding an exploding wire is shown in Fig. 3(e). In most cases, this shell was clearly visible with $30 \%-40 \%$ contrast. For the X-rays used for those imaging tests (3-5 keV), the contrast due solely to absorption would have been just a few percent, making the wire coating, even at solid density, very difficult to see [34]. It is possible to take advantage of such wave optics effects to image structure in objects that otherwise would be difficult to observe. As an example, we show in Fig. 4 a sample radiograph of a common domestic housefly. Numerous features in this image, such as the structure in the fly wings or the whiskers on the fly's nose, would be difficult to see without phase-contrast effects because the contrast ratios would be only $1 \%-2 \%$ [34].

Phase-contrast imaging and the high spatial resolution possible using $X$-pinch $\mathrm{X}$-ray sources make this type of imaging especially valuable for the study of biological objects. Work is currently in progress to study seed germination and plant structures in collaboration with agricultural researchers at Cornell University. 
(a)

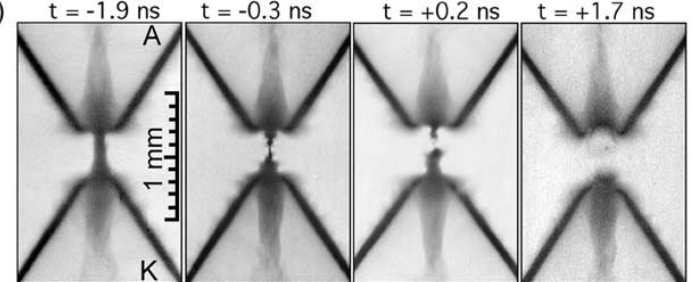

(b)

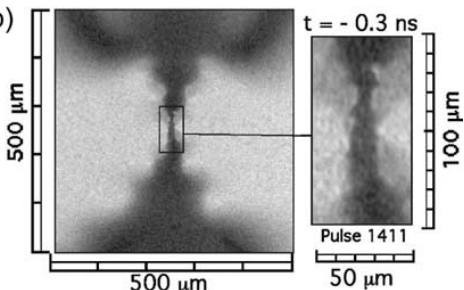

(c) $212.7 \mu \mathrm{m} \mathrm{Al}$ wires $\mathrm{t}=235 \mathrm{~ns}$

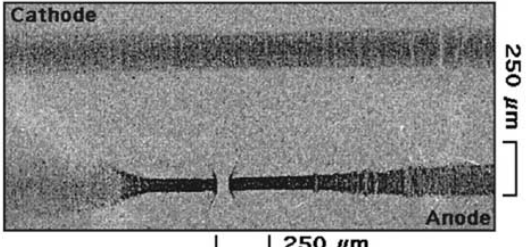

(d) $25 \mu \mathrm{m}$ Zn wire

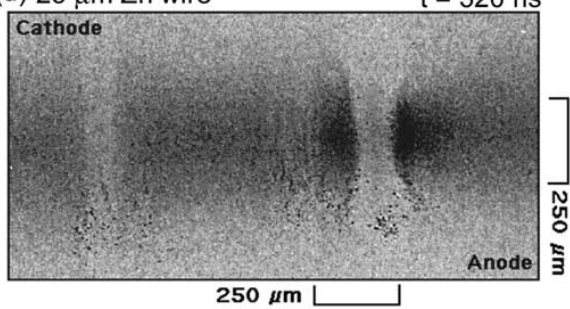

(e) $11.5 \mu \mathrm{m}$ W wire with a $2.2 \mu \mathrm{m}$ polyimide coating
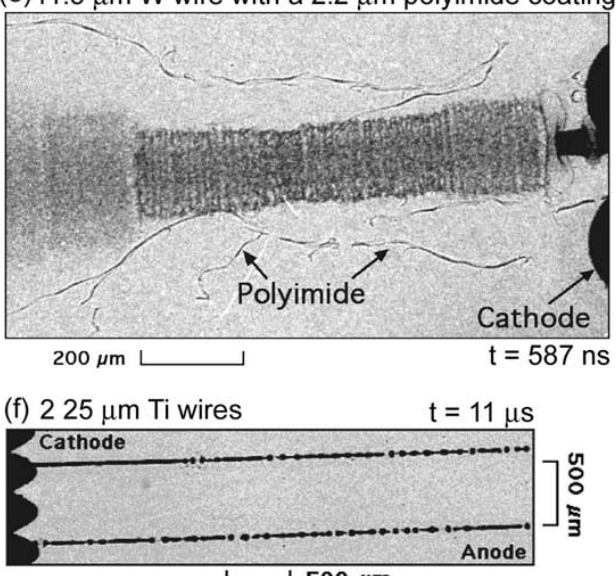

Fig. 3. (a) Sample radiographs showing the implosion of $17-\mu \mathrm{m}$-wire Mo $X$ pinches. The times shown above the images are relative to the time the $X$ pinch being imaged produced its first radiation burst. A miniature $Z$ pinch reliably forms at the cross point of the $X$ pinch, becomes unstable, and pinches toward the axis, producing an X-ray burst in the process, and finally breaks apart into a low-density plasma. (b) An expanded view of one of the frames, illustrating the narrow necks formed during the implosion. Experiments in [28] showed that the X-ray bursts are always emitted from these narrow neck regions. (c-f) Sample point-projection radiographs of exploding wires driven by a damped sinusoidal current pulse having a 350-ns quarter period rise time, an amplitude of $4.5 \mathrm{kA}$, and an $e$-fold damping time of $4.25 \mu \mathrm{s}$. The times shown are relative to the start of the current. These images illustrate the wide range of exploding-wire structure and behavior seen using the $X$-pinch backlighting method.

\section{FocUsing SPECTROGRAPHS With SPATIAL} RESOLUTION (FSSR) FOR THE 1-10-KEV RANGE

\section{A. FSSR-1D}

Spectroscopic diagnostics based on bent crystals have been routinely employed since the 1930s. Such diagnostics are based on the Bragg diffraction of X-rays from crystal planes. Only X-rays satisfying the Bragg condition $n \lambda=2 d \sin (\theta)$ are reflected by the crystal, where $\lambda$ is the incident photon wavelength, $d$ is the spacing of planes in the crystal, $\theta$ is the "grazing angle" (the angle with respect to the crystal plane), and $n$ is an integer corresponding to the crystal reflection order. Because the $2 d$ spacing of most crystals is in the 2-20- $\AA$ range, spectroscopic instruments using Bragg crystals are ideal for plasma spectroscopy in the $1-10-\mathrm{keV}(12-1.2-\AA)$ range.

During the 1970s, a modification of the Johann spectrograph was developed using spherically bent crystals instead of cylindrically bent crystals [10]. The geometry can be understood using a construct known as the Rowland circle. The Rowland circle has a diameter equal to the bending radius of the crystal and has the property that emission from a point on the Rowland circle is focused back to a point on the Rowland circle by a spherically bent crystal. In the plane of the
Rowland circle, referred to as the meridional plane, the instrument acts as an ordinary Johann spectrograph, dispersing the X-rays along the Rowland circle in accordance with the Bragg condition. In the plane perpendicular to the Rowland circle, referred to as the sagittal plane, the crystal acts as a mirror and focuses the X-rays in this plane. This setup is known as the Focusing Spectrograph with Spatial Resolution in 1-D (FSSR-1D). A schematic diagram of this arrangement is shown in Fig. 5.

The spatial resolution in this system depends on the spherical mirror optics and the fabrication quality of the crystal. One of the reasons these spectrographs were not feasible earlier was the difficulty of accurately bending large-aperture crystals with small bending radii. Spatial resolutions as good as $20 \mu \mathrm{m}$ have been claimed along the sagittal direction [10], [11].

The spectral resolution of the Johann crystal geometry is generally independent of the X-ray source size and depends primarily upon the angular dispersion properties of the crystal. Experimentally determined spectral resolutions $\lambda / \Delta \lambda=10^{4}-10^{5}$ have been measured using these types of instruments for near-normal angles of incidence. FSSR-1D diagnostics have been applied to numerous experiments studying the properties of bright spots in the $X$ pinch plasma [23], [24], [28] and the time history of the ion kinetic 
(a)

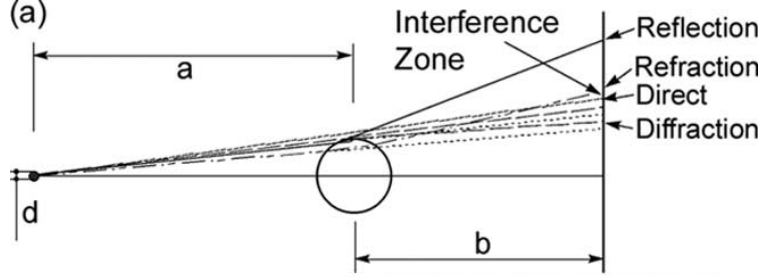

(b)

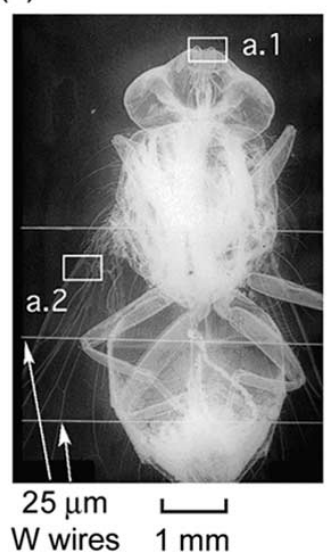

(c)

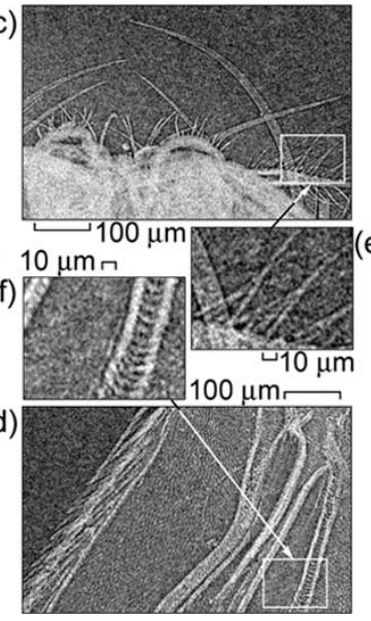

Fig. 4. (a) Diagram illustrating how wave optics effects can contribute to an image, thereby enhancing the contrast of an object above what is expected solely due to $\mathrm{X}$-ray attenuation. (b) Radiograph of a common housefly made using $X$-pinch point-projection backlighting. The features in this image would not have such high contrast if X-ray attenuation were the sole contributing factor to the image. (c) An expanded view of Fig. 4(b), area a.1, showing the whiskers on the fly's head. (d) An expanded view of Fig. 4(b), area a.2, showing the structure visible in the wings of the fly. (e), (f) Expanded views of boxed regions in Fig. 4(c) and (d), respectively.

energy in the gas-puff Z-pinch plasma [35]. Spectroscopic experiments using $X$ pinches have also enabled high-precision measurements of plasma ion line wavelengths to be measured [10], [20], [36]. Although this capability was developed at the Lebedev Institute and at Cornell University on modest-size pulsed power machines, the FSSR-1D configuration is very suitable for larger machines that might produce intense kiloelectronvolt X-ray spectra, such as the $\mathrm{Z}$ facility at Sandia National Laboratories.

\section{B. FSSR-2D}

In the traditional FSSR-1D configuration, the source is placed inside or outside the Rowland circle and the detector is placed on the Rowland circle. A slight variation of this geometry places both the source and the detector slightly outside the Rowland circle, as shown in Fig. 5. By placing the detector outside the Rowland circle, it is possible to make spectrally selective, 2-D images of the emitting plasma with spatial resolutions $<100 \mu \mathrm{m}$ along both directions. For nonnormal Bragg angles, however, the images created using this geometry can have relatively large optical distortions, making the images more difficult to analyze. As a result, this instrument has not been used extensively. Further details about this geometry can be found elsewhere [11], [37].

\section{One- To Ten-Kiloelectronvolt Monochromatic X-RAY IMAGING DiAGNOSTICS}

\section{A. Monochromatic X-Ray Microscopy Diagnostic}

During the early 1990s, it was recognized that 2-D, monochromatic self-emission images of plasmas could be obtained using bent crystals [12]. The self-emitting plasma is placed inside the Rowland circle. The detector is placed outside the Rowland circle at the focal position of the object, as determined using the spherical (or toroidal) lens equation(s). As in the spectroscopy geometries discussed in Section III, each point on the Rowland circle is associated with a wavelength, and the spectral bandpass of the imaging diagnostic is determined by the region of the Rowland circle intercepted by the reflected beam from the crystal. To minimize astigmatism, the Bragg angles are typically between $80^{\circ}$ and $90^{\circ}$ for spherical optics, though this range can be extended down to Bragg angles of $\sim 70^{\circ}$ through the use of toroidal optics. The microscopy geometry is illustrated in Fig. 6.

These systems are capable of $<10-\mu \mathrm{m}$ spatial resolution along both directions. This is because the geometry is fundamentally a point-to-point focusing configuration, in contrast to the more traditional pinhole camera imaging. The spectral bandpass of these systems is typically the same or slightly narrower than the width of the plasma spectral line used for imaging.

This geometry has been widely employed in recent years. Experiments at the Gekko laser facility, Osaka, Japan, used an array of toroidally bent $\mathrm{Si}$ and Ge crystals to obtain five gated, 2-D images of an Ar plasma in $\mathrm{Ar} \operatorname{Ly} \beta$ and $\mathrm{Ar} \mathrm{He} \beta$ line radiation, respectively [38]. Coupled with high-resolution X-ray spectroscopy, this microscopy diagnostic allowed a determination of time-dependent density gradients in an imploding Ar-doped, inertial-confinement-fusion capsule [39]. Lawrence Livermore National Laboratories is also actively investigating bent-crystal microscopy for similar applications on the National Ignition Facility [40].

\section{B. Monochromatic X-Ray Backlighting Diagnostic}

Using a variant of the bent-crystal microscopy geometry, shown schematically in Fig. 7, it is possible to obtain monochromatic backlighting images of dense plasma located inside the Rowland circle [13], [14]. In this case, the X-ray source is placed on the Rowland circle and the object is placed inside the Rowland circle. The spectral bandpass is then determined by the width of the $\mathrm{X}$-ray source on the Rowland circle. The spectral bandpass is usually much smaller than the width of the plasma line used for imaging, especially when the X-ray source size is small. The spatial resolution is also quite high. Spatial resolutions as small as $3 \mu \mathrm{m}$ have been reported using such a system with a magnification of 20 [41]. The field of view $F$ of these systems depends on the crystal aperture $L$ and the magnification $M$ and is given by $F=(L / 2)(1-1 / M)$ and can be very large, e.g., $4 \mathrm{~mm}$ by $20 \mathrm{~mm}$.

These systems have been applied at several laser facilities because of their high spatial resolution. For example, a 1.865-keV imaging system using the $\mathrm{Si} \mathrm{He}_{\alpha}$ line was developed for the NIKE laser facility at the Naval Research 

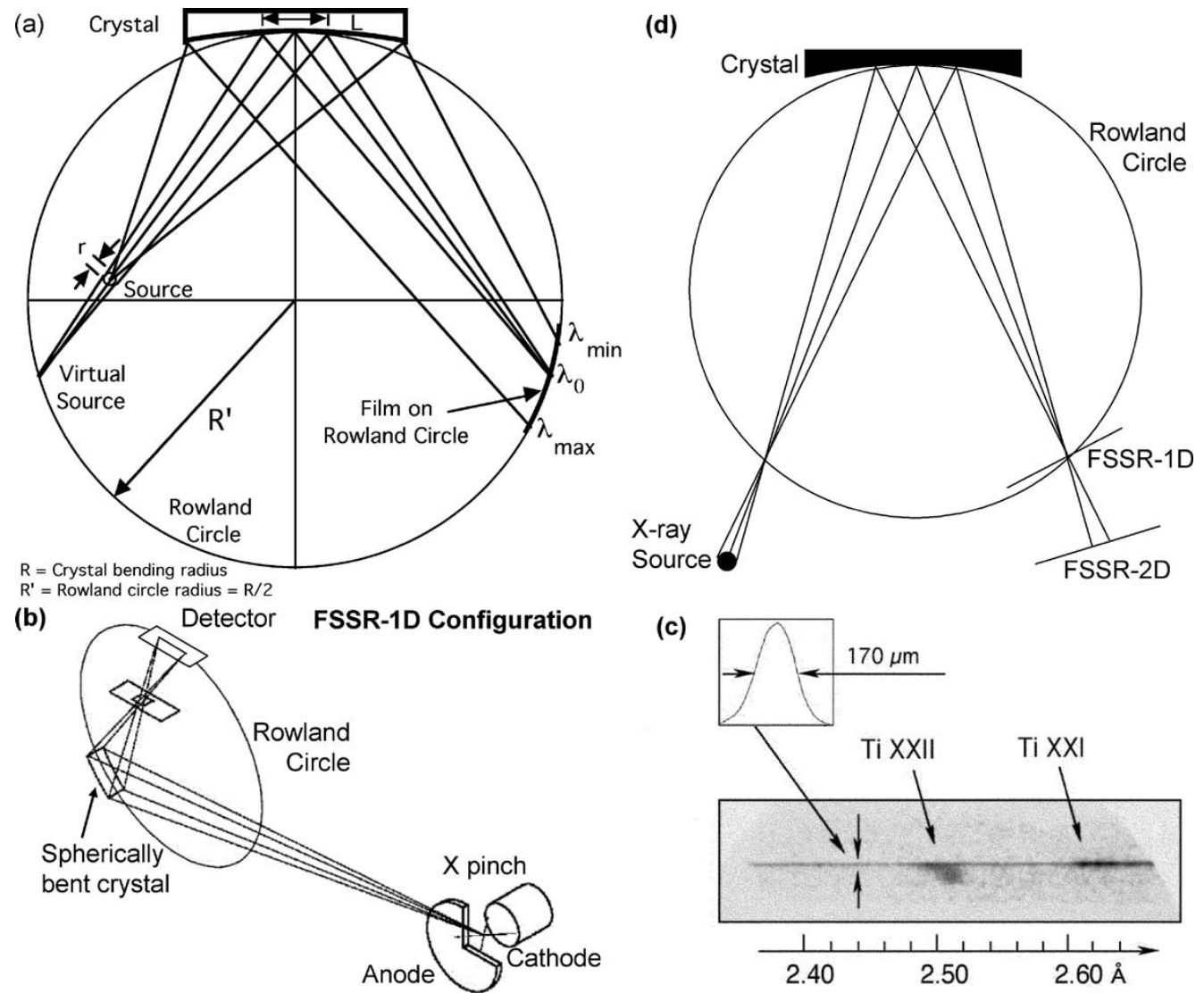

(c)

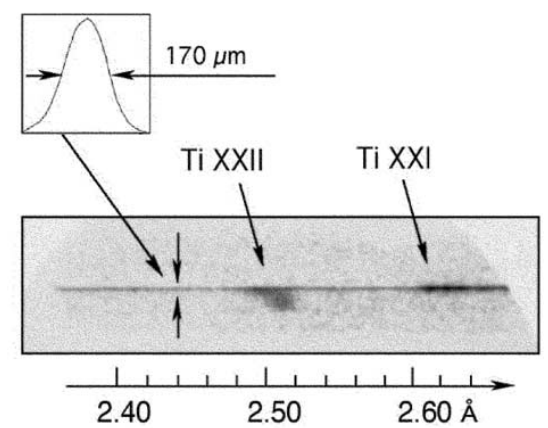

Fig. 5. (a) Diagram illustrating the Johann spectrograph geometry traditionally used with cylindrical crystals. (b) By bending the crystal along the plane perpendicular to the plane in Fig. 5(a) to form a spherically bent crystal, a focusing spectrograph with spatial resolution along the second dimension can be made [FSSR-1D configuration shown in Fig. 5(b)]. (c) A sample FSSR-1D image of Ti $X$-pinch radiation made using an X-ray streak camera photocathode. This is a static image, so the direction perpendicular to the spectral dispersion direction is spatially resolved. The continuum radiation can be seen to come from a much smaller spatial region than the line radiation. The $170-\mu \mathrm{m}$ continuum line width is the instrumental width of the streak camera system used to take this image. (d) By placing the detector outside the Rowland circle at the appropriate focal point, it is possible to form a 2-D image in monochromatic light as described in [37].
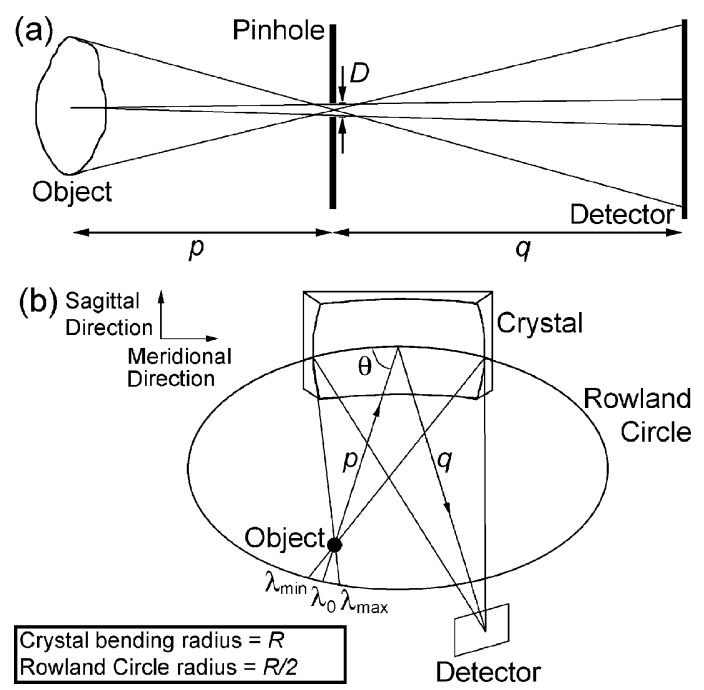

Fig. 6. (a) Schematic diagram of the traditional pinhole camera imaging diagnostic. The spatial resolution of this system is equal to or worse than the size of the pinhole. (b) Schematic diagram of a crystal microscope system. The detector is placed at the focal point of the object, so that the system is very nearly a point-to-point focusing geometry. In this way very high spatial resolutions are possible with good sensitivity using a high-quality bent-crystal reflector.

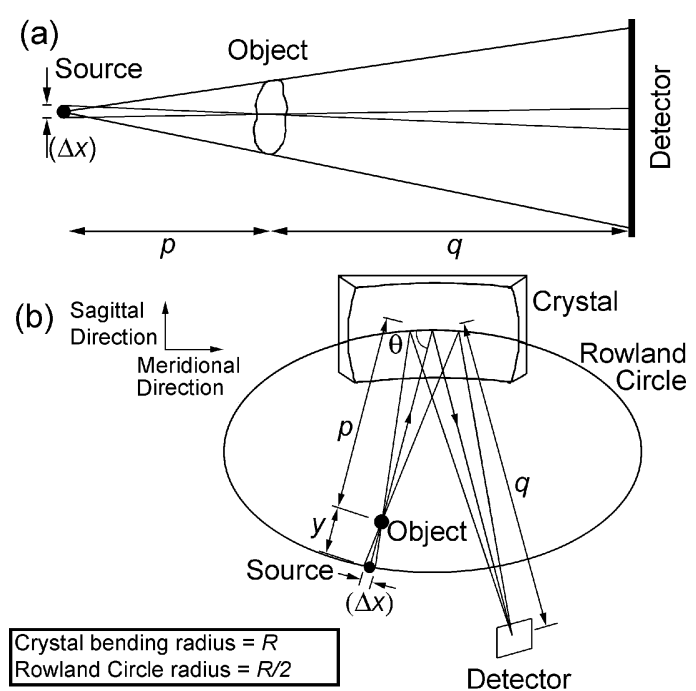

Fig. 7. (a) Schematic diagram of the traditional point-projection $\mathrm{X}$-ray backlighting geometry. The spatial resolution of this system depends on the magnification and the X-ray source size. (b) Schematic diagram of the crystal backlighting geometry. Like the crystal microscope, this system is very nearly a point-to-point focusing geometry and is capable of very high spatial resolutions for objects even at high magnification. The spatial resolution is very weakly dependent on the X-ray source size. 
(a) Pre-shot radiograph of a $20 \mathrm{~mm}$ diameter array with 300 8.7-micron diameter tungsten wires

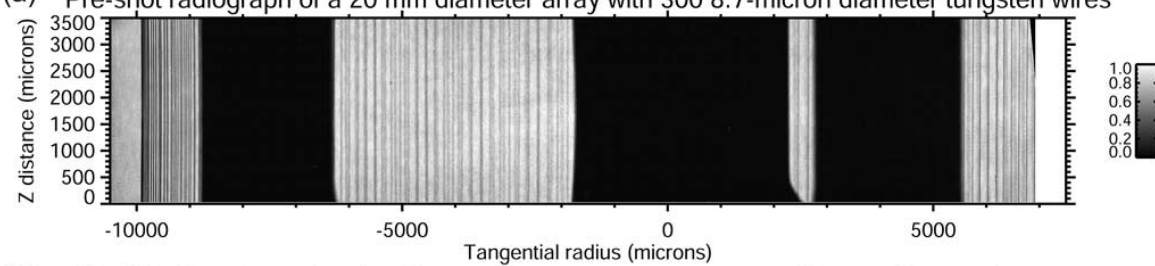

(b) "In flight" radiograph of a $20 \mathrm{~mm}$ diameter tungsten array $13 \mathrm{~ns}$ before peak $\mathrm{x}$ rays

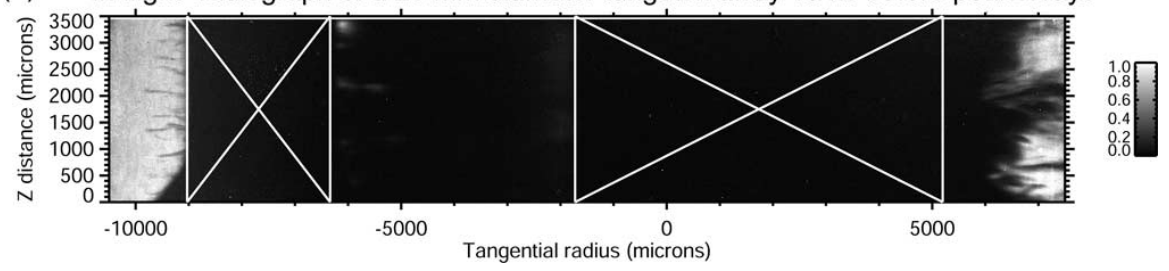

Fig. 8. (a) Example monochromatic (1.865-keV) radiograph of a static 20 -mm-diameter, cylindrical wire array of three hundred $8.7-\mu \mathrm{m}$ tungsten wires on the Sandia $\mathrm{Z}$ facility. The array is surrounded by a slotted return-current canister that partially blocks the view of the wire array, creating the three solid black regions in the image. The wires visible through the central slot opening appear wider and more transmissive because they are outside of the focal plane and are thereby imaged with a lower spatial resolution than wires near the tangential edge of the array. (b) Example monochromatic $(1.865-\mathrm{keV})$ radiograph of a similar wire array in motion as the $\mathrm{Z}$ facility current compresses the array mass toward the axis. These images illustrate the large fields of view possible using this technique and would not have been possible using point-projection backlighting because of the large debris and X-ray background produced by the wire-array implosion.

Laboratory, Washington, DC, for the study of planar instability growth [41]. A significant advantage of these systems for large-scale pulsed power facilities is their narrow spectral bandpass. Shortly after this diagnostic was developed, it was proposed for use on the Sandia $Z$ facility [42]. Wire-array $Z$-pinch implosions on the $\mathrm{Z}$ facility produce $\sim 1 \mathrm{MJ}$ of $\mathrm{X}$-rays in a quasi-blackbody spectrum with a high-energy tail. Traditional point-projection backlighting techniques are unsuitable for imaging the wire-array implosion because the object in that case is a strong source of debris and X-ray background. Using a $1.865-\mathrm{keV}$ crystal backlighter similar to that used on NIKE, radiograph images of wire arrays generating $\sim 1$ MJ of X-rays were made using a $\sim 1$-J X-ray source [43], [44]. An example image from such a test is presented in Fig. 8.

\section{Alternative Monochromatic Backlighting Geometries}

One of the drawbacks to the traditional monochromatic $\mathrm{X}$-ray backlighting geometry is the limitation that the Bragg angle is between $80^{\circ}$ and $90^{\circ}$. This requirement means that in order to use a given ion line for imaging, a crystal must be found with a $2 d$ spacing value (see Section III-A) that is within an integer multiple of $100 \%-105 \%$ of the emission line wavelength. This severely limits the possible lines that can be used as X-ray sources for crystal backlighting. This range can be extended down to Bragg angles of $\sim 70^{\circ}$ by using toroidally bent crystals, but such crystals must be fabricated exactly for the application in mind and have very stringent alignment requirements.

An alternative backlighting geometry was recently proposed that allows Bragg angles much less than $45^{\circ}$ to be used with spherically bent crystals [45]. This geometry is basically the same as the backlighting geometry in Section IV-B, but the detector is placed at a special compromise film position between the focal positions along the meridional and sagittal directions. In principle, it is possible to obtain $\sim 10-\mu \mathrm{m}$ spatial resolution along both directions using this geometry, but only if the X-ray source sizes are also quite small (10 $\mu \mathrm{m}$ or less).

Other variants of the traditional backlighting geometry in Section IV-B exist that place the object in different locations. In principle, high-resolution images can also be obtained when the objects are placed between the crystal and the detector, either before or after the focal point on the Rowland circle [46]. The images have noticeable distortions, but these can be calculated and so the image distortion can be removed computationally.

\section{UV-Light Plasma Diagnostics}

\section{A. Motivation for UV-Light Diagnostics}

Investigations of the properties of a $Z$-pinch plasma during the implosion phase are important because this stage of the $Z$-pinch experiments affects the plasma stagnation phase in which the X-ray output is generated. In particular, the magnetic field distribution plays a key role in determining the characteristics of the implosion and stagnation dynamics of the plasma, and theoretical models and simulations for Z-pinch plasmas strongly rely on assumptions on the magnetic field distribution. Knowledge of the history of the plasma parameters during the implosion allows for study of the energy terms in the plasma and is useful for developing and benchmarking radiation-hydrodynamic models.

Spectroscopic diagnostics in the UV regime have not been widely implemented in investigations of $Z$ pinches. Since the plasma is relatively cold and dilute during the implosion phase, X-ray self-emission diagnostics cannot be employed; therefore, UV diagnostics are advantageous for studying the plasma properties and dynamics prior to stagnation. In principle, these diagnostics can yield the histories of the magnetic field distribution in the imploding plasma, as well as the spatial distributions of the electron temperature, charge states, and ionic implosion velocities. It should be noted, however, 


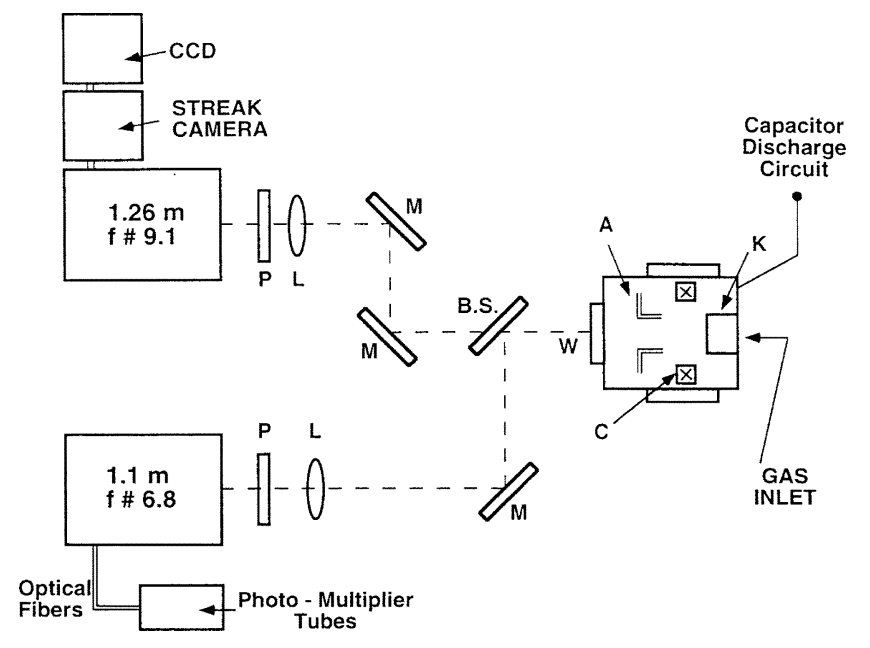

Fig. 9. A schematic of the WIS Z-pinch experiment and the diagnostic systems. $W, L, M$, and $B . S$. are a vacuum-chamber window, a lens, a mirror, and a beam splitter, respectively. The polarization of the collected emission is selected by the use of a rotatable $\lambda / 2$ plate $P$.

that spectroscopic investigations of the plasma during the implosion are complicated by the continuous flow of particles, the temporal and spatial dependence of the plasma properties, and the rapid-ionization processes in the plasma.

It is worth emphasizing that high-resolution UV diagnostics are suitable and promising both for small-scale university facilities, as well as for high-power $Z$-pinch machines (such as the Z facility [2] or Angara [3]). In particular, UV diagnostics are highly useful for studying the lower density plasmas left at larger radii during the implosion, the development of spatial nonuniformities and hydrodynamic instabilities, and the formation of shock waves in the imploding plasma.

The diagnostics described in the subsequent sections were developed and tested on the 220-kA, $\mathrm{CO}_{2}$ gas-puff $Z$-pinch facility at the Weizmann Institute [6], [47]. The typical implosion time of the plasma is about $620 \mathrm{~ns}$. A schematic of the facility is shown in Fig. 9. The diagnostic setup includes two UV spectroscopic systems, allowing for simultaneous observations of a few line emissions or different emission polarizations along either the $z$ axis (end-on), the radius (side-on), or various chords of the cylindrical plasma. The two systems include $1.26-\mathrm{m}$ and $1.1-\mathrm{m}$ spectrometers, both equipped with 2400-groove/mm gratings, yielding spectral resolutions of 0.05 and $0.07 \AA$, respectively. Recording instruments for these systems are a fast UV streak camera, lens-coupled to a charge-coupled device (CCD), and an array of $12 \mathrm{UV}$ photomultiplier tubes, respectively. For the magnetic field measurements, the input optics include a beam splitter and rotatable $\lambda / 2$ plates. The observed volume was typically $0.5 \mathrm{~mm}$ wide (in the radial direction) and $2.5 \mathrm{~mm}$ high (in the azimuthal direction). The temporal resolution chosen throughout this study is $10 \mathrm{~ns}$. Both systems are absolutely calibrated over the entire useful spectral range (2000-5000 ̊).

\section{B. Determination of the Magnetic Field Distribution}

The experimental technique and the results of the magnetic field measurements are described in detail in [6]. In brief, the method is based on using measurements of spectral shifts of
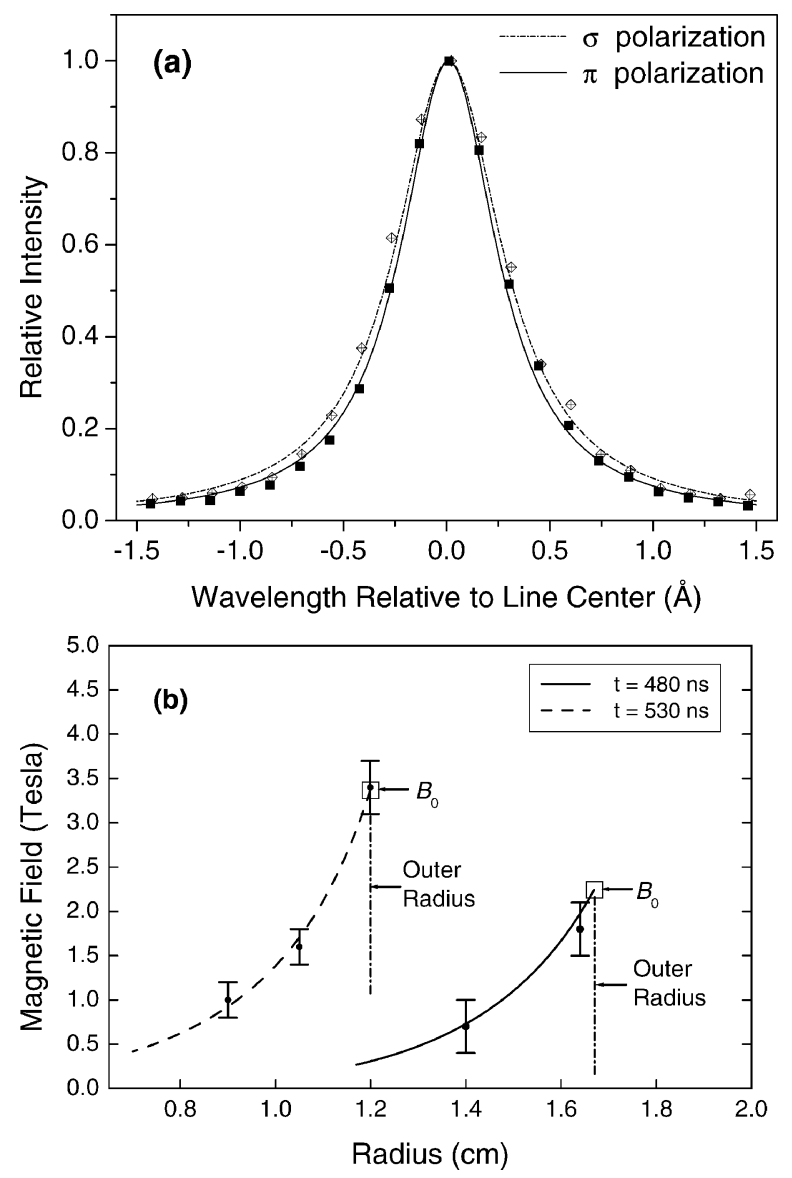

Fig. 10. (a) Measured profiles for the $\sigma$ and $\pi$ polarizations of the OIV 3063.5 - $\AA$ line for $t=480 \mathrm{~ns}$, each obtained by averaging 20 repeated discharges. The uncertainties are given by the size of the data points in the figure. The uncertainty in the FWHM is determined from the difference between the widths of profiles fitted to the upper or lower bounds of the data points. Here, $B_{\theta}$ obtained is $1.8 \pm 0.3 \mathrm{~T}$. (b) The magnetic field distribution in the plasma at $t=480$ and $t=535 \mathrm{~ns}$. The fitting curves for each time are based on the solution of a 1-D magnetic field diffusion equation. The boundary field $B_{0}$ is calculated from the known total discharge current and the outer radius of the plasma column (see [6]).

line emission from the plasma in the presence of a magnetic field (Zeeman splitting). In a Z-pinch plasma, the difficulties in such measurements arise from the fact that the splitting is small compared to the line widths, which for typical plasma parameters are dominated by Stark and Doppler broadening effects. In such a case, the difference in the spectral-shift pattern between the $\pi(\Delta m=0$, or linear) and the $\sigma(\Delta m= \pm 1$, or circular) polarization components of the lines can be used to determine the magnetic field strength. To accomplish that, end-on observations of line spectra from the $Z$ pinch using polarization optics were carried out, allowing for a comparison between the relative contributions of the $\pi$ and $\sigma$ components to the line widths and, thus, for discriminating the Zeeman splitting against other broadening mechanisms. The Stark broadening calculations used here are based on [48] and [49].

An example measurement is given in Fig. 10(a). Shown in the figure are the averaged profiles of the OIV $3 \mathrm{~s}-3 \mathrm{p}$ transition $(3063.47 \AA$ ), each obtained from 21 measurements at $r=1.65 \mathrm{~cm}$ and $t=480 \mathrm{~ns}$ for each polarization direction. The curves are best-fit Voigt profiles to the averaged 
data points, giving an uncertainty of $\pm 2 \%$ in the line FWHM. The fits shown give FWHM of $0.56 \pm 0.01$ and $0.62 \pm 0.01 \AA$ for the $\pi$ and $\sigma$ components, respectively. The comparison to the computer calculations of the line profiles for this example gives a magnetic field of $1.8 \pm 0.3 \mathrm{~T}$.

Following the same procedure for lines of OII-OVI observed at various radii and instants during the implosion, the time-dependent radial distribution of the field strength was obtained. For example, given in Fig. 10(b) are the radial distributions of $B_{\theta}$ for $t=480$ and $530 \mathrm{~ns}$. In the figure, the data-fitting curves are solutions of a 1-D magnetic diffusion equation, for which the plasma resistivity $\eta$ was used as a variable parameter. The resulting best-fit values of $\eta$ were found to agree reasonably well with Spitzer resistivity calculations made using the expected values of $T_{e}$ [6], [7]. The current density $J_{z}(r, t)$ is then obtained from the $B$-field distribution, which allows the $\vec{J} \times \vec{B}$ forces and the Joule heating rate to be calculated as a function of time and radial location.

It is interesting to note that the $B$ field, measured at the radially outermost region of the plasma, was found to be in excellent agreement (to within 5\%) with the value calculated from the total discharge current measured independently using a Rogowski loop, thus indicating that most of the circuit current flows within the imploding plasma shell.

\section{Determination of the Electron Temperature}

The time-dependent radial distribution of the electron temperature $T_{e}$ during the implosion, for times up to $50 \mathrm{~ns}$ before maximum compression, has been determined by comparison of the ionic level-population ratios of OII-OVI, obtained from end-on observations of line intensities, to the results of time-dependent collisional-radiative (C-R) calculations [50] in which $T_{e}$ is used as a variable input parameter. The $T_{e}$-sensitive ratios chosen for this study were independent of the electron density $n_{e}$ and the plasma composition history, which are usually not known with sufficient accuracy in transient, rapidly ionizing plasmas. For the expected conditions of the plasma under study, all lines chosen were optically thin.

The measurements were carried out for several radii and instants during the plasma inward acceleration, between $t=$ 440 to $570 \mathrm{~ns}$, in accordance with the charge-state radial distribution. In order to minimize the experimental uncertainties in the observed intensities, the data for each line were averaged over five to eight experiments, and two to four population ratios for each charge state were used. In the C-R analysis, the ratios were calculated for several values of $T_{e}$, and the range of $n_{e}$ chosen was based on continuum radiation measurements [51]. As an example, we present in Fig. 11 the time-dependent electron temperature at the outer plasma boundary, obtained from the analysis of level-population ratios of OIV-OVI. This plasma region was found to be the hottest and most dense part of the plasma. In this example, $T_{e}$ was determined both using population ratios within one charge state, and using ratios of levels from successive charge states, the latter with the proper detailed analysis [51], providing a higher accuracy.

Using the electron temperature obtained, together with the known time-dependent radial distributions of the ion directed velocities [47], magnetic field [6], and electron

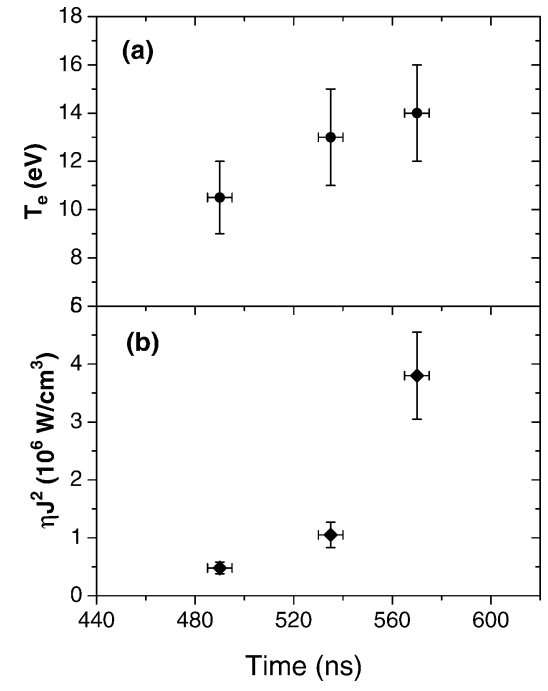

Fig. 11. (a) Electron temperature at the outer plasma boundary, obtained from measurements of line-intensity ratios of OIV-OVI, at three times during the plasma implosion. (b) Joule heating rate (per unit volume) calculated using the current density $J_{z}$ and the plasma resistivity $\eta$ at the outer plasma boundary for three times during the implosion.

density [51], all the energy and momentum deposition and dissipation terms in the plasma were calculated, allowing for a comparison between the magnetic energy delivered to the plasma to the energy acquired and dissipated by the plasma. The purpose of the energy-balance analysis is both to quantitatively determine the conversion efficiency of the capacitively stored energy into the kinetic energy of the plasma and to examine the consistency of all the results obtained for the plasma under study.

An example calculation of the time-dependent Joule heating rate at the plasma outer boundary is given in Fig. 10(b). Similar detailed calculations for all the energy terms were carried out for a time interval of $\sim 100 \mathrm{~ns}$ during the implosion phase, yielding the following findings [7]. Comparison between the energy deposition sources shows that the $\vec{J} \times \vec{B}$ forces provide about $85 \%$ of the total energy delivered to the plasma, while the Joule heating provides $\sim 15 \%$ of the energy. Calculations for the dissipation mechanisms show that $\sim 65 \%$ of the energy is acquired by plasma in the form of kinetic energy, $\sim 25 \%$ in the forms of heat and ionization, and $\sim 10 \%$ in the form of line and continuum radiation. The total deposited energy agrees well (to within $10 \%$ ) with the total delivered energy, thus demonstrating the consistency of the measurements described above.

The thermal pressure gradient, obtained from $n_{e}(r, t)$ and $T_{e}(r, t)$, allowed for the determination of the ion acceleration mechanisms across the plasma shell. It was found that only ions within 1-1.5 mm of the plasma outer boundary are accelerated by the magnetic field, while for the rest of the 5-mm-thick shell the ion momentum is gained via collisions. An additional important finding was the determination of the mechanisms supporting the radial propagation of the ionization front ahead of the plasma [47], where a nearly equal contribution from Joule heating, photoionization, and electron heat flux was obtained in the energy balance analysis for the plasma at the shell inner boundary. 


\section{Determination of the Electron Density}

In our plasma, the ion-velocity effects on the spectral line shapes were found to be of the same order of magnitude as the Stark line broadening, therefore not allowing the latter to be used as a sufficiently reliable method for the measurement of $n_{e}$ [51]. Therefore, in order to determine $n_{e}$ more accurately than the spatially averaged value obtained from the continuum measurements, two independent approaches were employed.

The first method is based on a self-consistent analysis of end-on observations of absolute line intensities from different charge states using C-R calculations, for which only knowledge of $T_{e}(r, t)$ and the system absolute calibration is required [52]. This analysis allows the absolute charge state densities in the plasma to be obtained using the following relation:

$$
N_{\mathrm{tot}}^{\alpha, j}=\frac{n_{u}^{\alpha, j}(\exp )}{\left[\frac{n_{u}^{\alpha, j}}{n_{\mathrm{tot}}^{\alpha}}\right]}
$$

Here, $n_{u}^{\alpha, j}$ and $n_{\text {tot }}^{\alpha}$ are the upper-level and total densities for the charge state $\alpha$ and the level $j$, obtained from the C-R calculations for a given $n_{e}$ and $T_{e}, n_{u}^{\alpha, j}(\exp )$ is the experimentally obtained upper-level population, and $N_{\mathrm{tot}}^{\alpha, j}$ is the total ion density for the charge state $\alpha$, calculated from the population of the upper-level $j$. The electron density associated with the oxygen ions is then obtained from $N_{e}=\sum_{\alpha} Z_{\alpha} N_{\text {tot }}^{\alpha}$, where $Z_{\alpha}$ is the ionic charge. This value is then compared to $n_{e}$ used in the C-R calculations. This procedure is repeated iteratively using various values of $n_{e}$ and the value for which a minimum in the value of $\delta n_{e}=\left(N_{e}-n_{e}\right) / n_{e}$ is reached is considered as the correct electron density. This analysis is then extended for cases of multispecies plasmas, such as in our case, where it also provides self-consistently the plasma composition and increases the accuracy in determining $T_{e}$ [52].

The second method is based on the comparison between the experimental and theoretical ionization times in the plasma [51], which requires the knowledge of $T_{e}(r, t)$, and the radial-distribution histories of the velocities and the charge states of the various ions. For the analysis, the plasma is divided into three concentric cylinders (mass cells), where for each region the time-dependent radial boundaries, the ion radial-velocity gradient, and $T_{e}(t)$ are all taken from experiment, and the plasma mass is treated as a variable, unknown parameter. A time-dependent C-R calculation for the mean charge $Z(t)$ is then carried out separately for each mass cell, accounting for the radial displacement of the plasma in the cell and the mass transfer into or from the neighboring cells due to the differences between the directed radial velocities of ions and the propagation velocity of the ionization [47]. This procedure is carried out iteratively using various input values of $n_{e}$. The values which give the best agreement between the calculated values of $Z(t)$ and the values observed experimentally, are then considered as the correct electron density for each cell.

The above two methods agree to within $\sim 20 \%$ in the value of the mass line-density obtained. The mean result, $7.5 \pm$
$2.5 \mu \mathrm{g} / \mathrm{cm}$, agrees to within $\sim 30 \%$ with the independently measured initial radial distribution of the gas density. This shows the reliability of the spectroscopic methods, and also indicates that most of the initial plasma mass is involved in the implosion process [51].

\section{SUMMARY}

This review paper merely highlights some of the important research and diagnostic developments that have taken place on small-scale pulsed power facilities. We have chosen to limit these highlights to only those dealing with X-ray and UV diagnostics, though excellent work has been carried out in recent years using optical diagnostics as well. Examples include schlieren imaging and optical interferometry of exploding-wire $Z$-pinch implosions that have been carried out on their 1-MA pulser, MAGPIE [53], [54], optical interferometry measurements of plasma jets in $X$ pinches made on the 100-kA Gepopu generator, Santiago, Chile [55], and optical Faraday rotation measurements also carried out on MAGPIE [56]. Many of the diagnostic methods discussed in this paper have already or soon will be applied on major facilities, including the Sandia $\mathrm{Z}$ facility and the National Ignition Facility. In this way, the research conducted on these smaller facilities can be considered to have directly contributed to work on the larger facilities.

In addition to the direct contributions by way of diagnostic development, the experiments highlighted here studying the $X$-pinch $\mathrm{X}$-ray sources and gas-puff $Z$-pinch sources have contributed to our fundamental understanding of $Z$-pinch plasma behavior. Though $X$-pinch plasmas have been routinely used as X-ray sources for over two decades, the mechanisms and plasma dynamics responsible for the production of the bright spots seen in $X$ pinches are still not well understood, and research in this area is ongoing as more sophisticated diagnostics and spectroscopic models are developed, e.g., [57], [58]. Similarly, though $Z$ pinches have been studied since the beginning of plasma physics, mid- and high- $Z$ material $Z$ pinches still defy simulations and calculations because of the complex physical processes and multiple scale lengths involved. For example, photoionization and heat transport processes are known to take place in $X$ pinches, and represent an area where small machines may offer opportunities to develop and test models that may help understand the same processes in larger high energy density facilities. Thus, university researchers in pulsed power are still contributing not only at the level of diagnostic development but also at the level of fundamental science.

\section{ACKNOWLEDGMENT}

The authors wish to acknowledge the importance to this work of the collaboration on $X$-pinch physics during the past ten years between Cornell University, Ithaca, NY, and the P. N. Lebedev Physical Institute, Moscow, Russia. This collaboration, originally organized by Dr. A. Lebedev, has had as its principal collaborators Dr. S. Pikuz and Dr. T. Shelkovenko, both of whom were instrumental in the development of X-ray-based research methods at Cornell 
University. The authors would also like to thank E. Kroupp for his significant contribution to the measurements, Dr. A. Fisher for his continuous help in the experimental work on the Z-pinch facility at the Weizmann Institute, Rehovot, Israel, and Dr. Y. Ralchenko for his support with the atomic physics and line broadening calculations.

\section{REFERENCES}

[1] J. Lindl, P. Amendt, R. L. Berger, S. G. Glendinning, S. H. Glenzer, S. W. Haan, R. L. Kauffman, O. L. Landen, and L. J. Suter, "The physics basis for ignition using indirect-drive targets on the National Ignition Facility," Phys. Plasmas, vol. 11, pp. 339-491, 2004.

[2] R. B. Spielman et al., "Tungsten wire-array Z-pinch experiments at 200 TW and 2 MJ," Phys. Plasmas, vol. 5, pp. 2105-2111, 1998.

[3] V. V. Alexandrov, I. N. Frolov, M. V. Fedulov, E. V. Grabovsky, K. N. Mitrofanov, S. L. Nedoseev, G. M. Oleinik, I. Y. Porofeev, A. A. Samokhin, P. V. Sasorov, V. P. Smirnov, G. S. Volkov, M. M. Zurin, and G. G. Zukakischvili, "Prolonged plasma production at currentdriven implosion of wire arrays on Angara-5-1 facility," IEEE Trans. Plasma Sci., vol. 30, pp. 559-566, Apr. 2002.

[4] T. R. Boehly, D. L. Brown, R. S. Craxton, R. L. Keck, J. P. Knauer, J. H. Kelly, T. J. Kessler, S. A. Kumpan, S. J. Loucks, S. A. Letzring, F. J. Marshall, R. L. McCrory, S. F. B. Morse, W. Seka, J. M. Soures, and C. P. Verdon, "Initial performance results of the OMEGA laser system," Opt. Commun., vol. 133, pp. 495-506, 1997.

[5] N. R. Pereira and J. Davis, "X-rays from z-pinches on relativistic electron-beam generators," J. Appl. Phys., vol. 64, pp. R1-R27, 1988.

[6] G. Davara, L. Gregorian, E. Kroupp, and Y. Maron, "Spectroscopic determination of the magnetic field distribution in an imploding plasma," Phys. Plasmas, vol. 5, pp. 1068-1075, 1998.

[7] L. Gregorian, G. Davara, E. Kroupp, V. A. Bernshtam, A. Starobinets, Y. V. Ralchenko, and Y. Maron, "Electron temperature and energy flow history in an imploding plasma," Weizmann Inst., Rep. No. WIS/22/02-June-DPP, 2002

[8] S. M. Zakharov, G. V. Ivanenkov, A. A. Kolomenskii, S. A. Pikuz, A. I. Samokhin, and J. Ulshmid, "Wire $X$ pinch in a high-current diode," Sov. Tech. Phys. Lett., vol. 8, pp. 456-457, 1982.

[9] D. H. Kalantar and D. A. Hammer, "The $X$ pinch as a point source of X-rays for backlighting," Rev. Sci. Instrum., vol. 66, pp. 779-781, 1995.

[10] V. A. Boiko, A. V. Vinogradov, S. A. Pikuz, I. Y. Skobelev, and A. Y. Faenov, "X-ray spectroscopy of laser-produced plasma," J. Sov. Laser Res., vol. 6, pp. 85-290, 1985.

[11] I. Y. Skobelev, A. Y. Faenov, B. A. Bryunetkin, V. M. Dyakin, T. A. Pikuz, S. A. Pikuz, T. A. Shelkovenko, V. M. Romanova, and A. R. Mingaleev, "Investigating the emission properties of plasma structures with X-ray imaging spectroscopy," JETP, vol. 81, pp. 692-718, 1995.

[12] E. Forster, K. Gabel, and I. Uschmann, "X-ray microscopy of laserproduced plasmas with the use of bent crystals," Laser Part. Beams, vol. 9, pp. 135-148, 1991.

[13] S. A. Pikuz, T. A. Shelkovenko, V. M. Romanova, D. A. Hammer, A. Y. Faenov, V. A. Dyakin, and T. A. Pikuz, "Monochromatic X-ray probing of an ultradense plasma," JETP Lett., vol. 61, pp. 638-644, 1995.

[14] — "High-luminosity monochromatic X-ray backlighting using an incoherent plasma source to study extremely dense plasmas (invited)," Rev. Sci. Instrum., vol. 68, pp. 740-744, 1997.

[15] D. Mosher, S. J. Stephanakis, L. S. Levine, D. J. Nagel, I. M. Vitkovitsky, and C. M. Dozier, "X-radiation from high energy density exploded wire discharges," Appl. Phys. Lett., vol. 23, pp. 429-430, 1973.

[16] P. G. Burkhalter, C. M. Dozier, and D. J. Nagel, "X-ray spectra from exploded-wire plasmas," Phys. Rev. A, vol. 15, pp. 700-717, 1977.

[17] D. A. Hammer, D. H. Kalantar, K. C. Mittal, and N. Qi, "X-pinch soft X-ray source for microlithography," Appl. Phys. Lett., vol. 57, pp. 2083-2085, 1990.

[18] D. H. Kalantar, D. A. Hammer, K. C. Mittal, N. Qi, J. R. Maldonado, and Y. Vladmirsky, "Intense pulsed plasma X-ray source for lithography," J. Vac. Sci. Technol. B, vol. 9, pp. 3245-3249, 1991.

[19] S. C. Glidden, M. R. Richter, D. A. Hammer, and D. H. Kalantar, "1 kW X-pinch soft X-ray source," Proc. SPIE, vol. 2194, pp. 209-220, 1994.
[20] A. L. Osterheld, A. I. Magunov, V. M. Dyakin, A. Y. Faenov, T. A. Pikuz, I. Y. Skobelev, T. Pisarczyk, P. Parys, J. Wolowski, J. Makowski, S. A. Pikuz, V. M. Romanova, and T. A. Shelkovenko, "Measurements of the ground-state ionization energy and wavelengths for the $1 \mathrm{~s}^{2}{ }^{1} \mathrm{~S}_{0}-1 \mathrm{snp}{ }^{1} \mathrm{P}_{1}(\mathrm{n}=6-12)$ lines of $\mathrm{Al}$ XII," Phys. Rev. A, vol. 54, pp. 3971-3976, 1996.

[21] S. A. Pikuz, B. A. Bryunetkin, G. V. Ivanenkov, A. R. Mingaleev, V. M. Romanova, I. Y. Skobelev, A. Y. Faenov, S. Y. Khakhalin, and T. A. Shelkovenko, "Radiative properties of a hot dense $X$ pinch," $J$. Quant. Spectrosc. Radiat. Transf., vol. 51, pp. 291-302, 1994.

[22] T. A. Shelkovenko, D. B. Sinars, S. A. Pikuz, K. M. Chandler, and D. A. Hammer, "Point-projection X-ray radiography using an $X$ pinch as the radiation source," Rev. Sci. Instrum., vol. 72, pp. 667-670, 2001.

[23] S. A. Pikuz, D. B. Sinars, T. A. Shelkovenko, K. M. Chandler, D. A. Hammer, G. V. Ivanenkov, W. Stepniewski, and I. Y. Skobelev, "High energy density $Z$-pinch plasma conditions with picosecond time resolution," Phys. Rev. Lett., vol. 89, pp. 035 003-1-035 003-4, 2002.

[24] D. B. Sinars, S. A. Pikuz, T. A. Shelkovenko, K. M. Chandler, D. A. Hammer, and J. P. Apruzese, "Time-resolved spectroscopy of Al, Ti, and Mo $X$ pinch radiation using an X-ray streak camera," J. Quant. Spectrosc. Radiat. Transf., vol. 78, pp. 61-83, 2003.

[25] G. V. Ivanenkov and W. Stepniewski, "Three-temperature model for the dynamics of a plasma produced by exploding metal wires," Plasma Phys. Rep., vol. 26, pp. 21-32, 2000.

[26] — , "MHD simulations of molybdenum $X$ pinches," Plasma Phys. Rep., vol. 28, pp. 886-897, 2002.

[27] D. H. Kalantar and D. A. Hammer, "Observation of a stable dense core within an unstable coronal plasma in wire-initiated dense Z-pinch experiments," Phys. Rev. Lett., vol. 71, pp. 3806-3809, 1993.

[28] T. A. Shelkovenko, D. B. Sinars, S. A. Pikuz, and D. A. Hammer, "Radiographic and spectroscopic studies of $X$-pinch plasma implosion dynamics and X-ray burst emission characteristics," Phys. Plasmas, vol. 8, pp. 1305-1318, 2001.

[29] D. B. Sinars, M. Hu, K. M. Chandler, T. A. Shelkovenko, S. A. Pikuz, J. B. Greenly, D. A. Hammer, and B. R. Kusse, "Experiments measuring the initial energy deposition, expansion rates and morphology of exploding wires with about $1 \mathrm{kA}$ /wire," Phys. Plasmas, vol. 8, pp. 216-230, 2001.

[30] S. V. Lebedev, F. N. Beg, S. N. Bland, J. P. Chittenden, A. E. Dangor, M. G. Haines, M. Zakullah, S. A. Pikuz, T. A. Shelkovenko, and D. A. Hammer, "X-ray backlighting of wire array Z-pinch implosions using $X$ pinch," Rev. Sci. Instrum., vol. 72, pp. 671-673, 2001.

[31] F. N. Beg, K. Krushelnick, P. Lichtsteiner, A. Meakins, A. Kennedy, N. Kajumba, G. Burt, and A. E. Dangor, "Table-top $X$ pinch for radiography," Appl. Phys. Lett., vol. 82, pp. 4602-4604, 2003.

[32] A. Snigirev, I. Snigireva, V. Koch, S. Kuznetsov, and Schelokov, "On the possibilities of X-ray phase contrast microimaging by coherent high-energy synchrotron radiation," Rev. Sci. Instrum., vol. 66, pp. 5486-5492, 1995.

[33] S. W. Wilkins, T. E. Gureyev, D. Gao, A. Pogany, and A. W. Stevenson, "Phase-contrast imaging using polychromatic hard X-rays," Nature, vol. 384, pp. 335-338, 1996.

[34] S. A. Pikuz, T. A. Shelkovenko, D. B. Sinars, K. M. Chandler, and D. A. Hammer, "Phase-contrast $X$-ray radiography using $X$ pinch radiation," in Proc. SPIE, vol. 4504, 2001, pp. 234-239.

[35] E. Kroupp, D. Carasso, D. Osin, A. Starobinets, V. Bernshtam, V. Fisher, Y. V. Ralchenko, Y. Zarnitsky, Y. Maron, I. Uschmann, E. Foerster, and A. Fisher, "High-resolution spectroscopic X-ray diagnostics for studying the ion kinetic energy and plasma properties in a $Z$ pinch at stagnation," Dig. Tech. Papers 14th IEEE Int. Pulsed Power Conf., pp. 209-212, 2003.

[36] A. R. Mingaleev, S. A. Pikuz, V. M. Romanova, T. A. Shelkovenko, A. Y. Faenov, and S. A. Ermatov, "Spectra of multiply charged nickel and copper ions in an X-pinch plasma," Quantum Electron., vol. 23, pp. 397-405, 1993.

[37] T. A. Pikuz, A. Y. Faenov, S. A. Pikuz, V. M. Romanova, and T. A. Shelkovenko, "Bragg X-ray optics for imaging spectroscopy of plasma microsources," J. X-Ray Sci. Tech., vol. 5, pp. 323-340, 1995.

[38] I. Uschmann, K. Fujita, I. Niki, R. Butzbach, H. Nishimura, J. Funakura, M. Nakai, E. Forster, and K. Mima, "Time-resolved ten-channel monochromatic imaging of inertial confinement fusion plasmas," Appl. Opt., vol. 39, pp. 5865-5871, 2000. 
[39] I. Golovkin, R. Mancini, S. Louis, Y. Ochi, K. Fujita, H. Nishimura, H. Shirga, N. Miyanaga, H. Azechi, R. Butzbach, I. Uschmann, E. Forster, J. Delettrez, J. Koch, R. W. Lee, and L. Klein, "Spectroscopic determination of dynamic plasma gradients in implosion cores," Phys. Rev. Lett., vol. 88, pp. 045 002-1-045 002-4, 2002.

[40] J. A. Koch, O. L. Landen, T. W. Barbee, P. Celliers, L. B. DaSilva, S. G. Glendinning, B. A. Hammel, D. H. Kalantar, C. Brown, J. Seely, G. R. Bennett, and W. Hsing, "High-energy X-ray microscopy techniques for laser-fusion plasma research at the national ignition facility," Appl. Opt., vol. 37, pp. 1784-1795, 1998.

[41] Y. Aglitskiy, T. Lehecka, S. Obenschain, S. Bodner, C. Pawley, K. Gerber, J. Sethian, C. M. Brown, J. Seely, U. Feldman, and G. Holland, "High-resolution monochromatic X-ray imaging system based on spherically bent crystals," Appl. Opt., vol. 37, pp. 5253-5261, 1998.

[42] S. A. Pikuz, T. A. Shelkovenko, D. A. Hammer, and D. F. Acton, "Monochromatic X-ray backlighting for application to PBFA-Z," in AIP Conf. Proc., Dense Z Pinches, vol. 409, 1997, pp. 523-526.

[43] D. B. Sinars, G. R. Bennett, D. F. Wenger, M. E. Cuneo, and J. L. Porter, "Evaluation of bent-crystal X-ray backlighting and microscopy techniques for the Sandia Z machine," Appl. Opt., vol. 42, pp. 4059-4071, 2003.

[44] D. B. Sinars, M. E. Cuneo, G. R. Bennett, D. F. Wenger, L. E. Ruggles, M. F. Vargas, J. L. Porter, R. G. Adams, D. W. Johnson, K. L. Keller, P. K. Rambo, D. C. Rovang, H. Seamen, W. W. Simpson, I. C. Smith, and S. C. Speas, "Monochromatic X-ray backlighting of wire-array $Z$-pinch plasmas using spherically bent quartz crystals," Rev. Sci. Instrum., vol. 74, pp. 2202-2205, 2003.

[45] M. S. del Rio, L. Alianelli, T. A. Pikuz, and A. Y. Faenov, "A novel imaging X-ray microscope based on a spherical crystal," Rev. Sci. Instrum., vol. 72, pp. 3291-3303, 2001.

[46] A. Y. Faenov, private communication, Apr. 2003.

[47] M. E. Foord, Y. Maron, G. Davara, L. Gregorian, and A. Fisher, "Particle velocity distributions and ionization processes in a gas-puff $Z$ pinch," Phys. Rev. Lett., vol. 72, pp. 3827-3830, 1994.

[48] S. Alexiou and Y. Maron, "Theoretically based closed form formulas for the collision operator for isolated ion lines in the standard starkbroadening theory," J. Quant. Spectrosc. Radiat. Transf., vol. 53, pp. 109-124, 1995.

[49] H. R. Griem, Y. V. Ralchenko, and I. Bray, "Stark broadening of the B III 2s-2p lines," Phys. Rev. E, vol. 56, pp. 7186-7192, 1997.

[50] Y. V. Ralchenko and Y. Maron, "Accelerated recombination due to resonant deexcitation of metastable states," J. Quant. Spectrosc. Radiat. Transf., vol. 71, pp. 609-621, 2001.

[51] L. Gregorian, G. Davara, E. Kroupp, V. I. Fisher, A. Starobinets, V. A. Bernshtam, and Y. Maron, "Plasma density and ionization dynamics in an imploding Z-pinch plasma," Weizmann Inst., Rep. No. WIS/23/02-June-DPP, 2002.

[52] L. Gregorian, V. A. Bernshtam, E. Kroupp, G. Davara, and Y. Maron, "Use of emission-line intensities for a self-consistent determination of the particle densities in a transient plasma," Phys. Rev. E, vol. 67, pp. 016 404-1-016 404-6, 2003.

[53] S. V. Lebedev, I. H. Mitchell, R. Aliaga-Rossel, S. N. Bland, J. P. Chittenden, A. E. Dangor, and M. G. Haines, "Azimuthal structure and global instability in the implosion phase of wire array Z-pinch experiments," Phys. Rev. Lett., vol. 81, pp. 4152-4155, 1998.

[54] S. V. Lebedev, F. N. Beg, S. N. Bland, J. P. Chittenden, A. E. Dangor, M. G. Haines, S. A. Pikuz, and T. A. Shelkovenko, "Effect of corecorona plasma structure on seeding of instabilities in wire array $Z$ pinches," Phys. Rev. Lett., vol. 85, pp. 98-101, 2000.

[55] I. H. Mitchell, R. Aliaga-Rossel, R. Saavedra, H. Chuaqui, M. Favre, and E. S. Wyndham, "Investigation of the plasma jet formation in $X$-pinch plasmas using laser interferometry," Phys. Plasmas, vol. 7, pp. $5140-5147,2000$.

[56] M. Tatarakis, R. Aliaga-Rossel, A. E. Dangor, and M. G. Haines, "Optical probing of fiber Z-pinch plasmas," Phys. Plasmas, vol. 5, pp. 682-691, 1998.

[57] A. S. Shlyaptseva, S. B. Hansen, V. L. Kantsyrev, D. A. Fedin, N. Ouart, K. B. Fournier, and U. I. Safranova, "Advanced spectroscopic analysis of 0.8-1.0 MA Mo $X$ pinches and the influence of plasma electron beams on L-shell spectra of Mo ions," Phys. Rev. E, vol. 67, pp. 026 409-1-026 409-9, 2003.

[58] A. S. Shlyaptseva, D. A. Fedin, S. M. Hamasha, S. B. Hansen, C. Harris, V. L. Kantsyrev, P. Neill, N. Ouart, P. Beiersdorfer, and U. I. Safranova, "X-ray spectroscopy and spectropolarimetry of high energy density plasma complemented by LLNL electron beam ion trap experiments," Rev. Sci. Instrum., vol. 74, pp. 1947-1950, 2003.

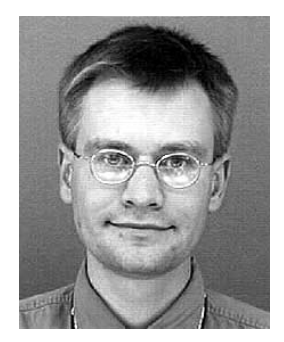

Daniel B. Sinars received the B.S. degree in engineering physics from the University of Oklahoma, Norman, and the Ph.D. degree in applied physics from Cornell University, Ithaca, NY, in 1996 and 2001, respectively. His thesis topic was time-resolved measurements of plasma conditions in $X$-pinch plasmas using $\mathrm{X}$-ray spectroscopy.

$\mathrm{He}$ is currently a Senior Member of the technical staff, Sandia National Laboratories, Albuquerque, NM. His research interests while at Cornell included experiments studying the explosion of single wires and $X$ pinches. His present research interests include dense $Z$-pinch physics experiments on the 20-MA Sandia Z pulsed power facility, where he has been implementing X-ray backlighting diagnostics using some of the monochromatic backlighting techniques discussed in this paper.

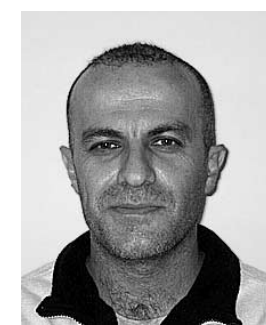

density histories.

Lev Gregorian was born in Moscow, Russia, in 1963. He received the M.Sc. degree in physics from the Weizmann Institute of Science, Rehovot, Israel, in 1993. He is currently working toward the Ph.D. degree in physics at the Weizmann Institute of Science.

His main research field has been the study of plasma dynamics and ionization processes in a z-pinch. This research is performed using spatially resolved UV spectroscopic measurements of the magnetic field, electron temperature, and

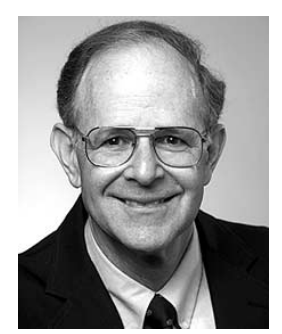

David A. Hammer (Fellow, IEEE) received the B.S. degree in physics from the California Institute of Technology, Pasadena, in 1964 and the Ph.D. degree in applied physics from Cornell University, Ithaca, NY, in 1969. His dissertation was on the topic of intense relativistic electron beam propagation.

After seven years at the Naval Research Laboratory, Washington, DC, and one year at the University of California, Los Angeles, he returned to Cornell University as a faculty member. He is currently the J. Carlton Ward Professor of Nuclear Energy Engineering and a Professor of Electrical and Computer Engineering. He has coauthored approximately 100 published papers in refereed scientific journals. His research interests included pulsed power-based plasma studies, including intense electron and ion beams and dense $Z$ pinches. In recent years, he has concentrated on the physics of exploding-wire plasma initiation and the dynamics of dense $Z$-pinch implosions.

Dr. Hammer is a Fellow of the American Physical Society and the American Association for the Advancement of Science, and is a Member of Sigma $\mathrm{Xi}$ and the University Fusion Association.

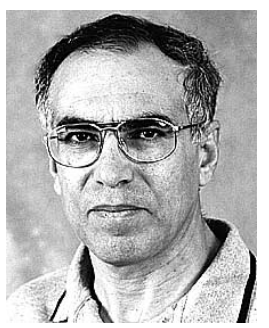

Yitzhak Maron (Member, IEEE) received the $\mathrm{Ph} . \mathrm{D}$. degree in physics from the Weizmann Institute of Science, Rehovot, Israel, in 1977

From 1980 to 1984, he worked on ion diodes at the Laboratory of Plasma Studies, Cornell University, Ithaca, NY. Since 1988, he has been a Professor of Physics and the Head of the Plasma Laboratory, Faculty of Physics, Weizmann Institute of Science, which specializes in the use of spectroscopy in the diagnostics of the properties of the plasmas and of the electric-magnetic fields in pulsed power systems, as well as in developing atomic physics models required for the interpretation of data from hot and dense plasmas. His activities encompass electron and ion diodes, plasma switches, and Z-pinches.

Prof. Maron is a Fellow of the American Physical Society. 